NUC-MINN-99/7-T

\title{
Baryon Masses in Chiral Perturbation Theory with Infrared Regularization
}

\author{
P. J. Ellis and K. Torikoshi \\ School of Physics and Astronomy, University of Minnesota \\ Minneapolis, MN 55455
}

(September 11, 2018)

\begin{abstract}
The baryon masses are examined in $S U(3)$ chiral perturbation theory to third order using the recently proposed infrared regularization scheme. Fourth order is estimated by evaluating the dominant diagram. With this regularization the magnitude of the loop integrals is reduced so that the convergence of the series appears to be better than in the heavy baryon approach.
\end{abstract}

PACS number(s): 12.39.Fe, 14.20.-c

Typeset using REVTEX 


\section{INTRODUCTION}

The description of the masses of the baryon octet in $S U(3)$ chiral perturbation theory is not in a satisfactory state. Using heavy baryon chiral perturbation theory (HBChPT) Borasoy and Meissner [1] have carried calculations to order $Q^{4}$, where $Q$ denotes a small momentum or a meson mass. The contributions of order $Q^{2}, Q^{3}$ and $Q^{4}$ are of roughly similar magnitude with alternating sign, thus casting doubt on the convergence of the expansion. Donoghue and Holstein [2] have suggested that the loop integrals should be regularized by introducing form factors. While this can be used to make the loop contributions tiny, it has the disadvantage of being model dependent and it is difficult to see how to apply it

consistently in general situations. An alternative approach has recently been suggested by Becher and Leutwyler [3] following the work of Ref. [4]. It employs chiral perturbation theory in manifestly Lorentz invariant form with the loop integrals evaluated in the socalled infrared regularization (IR) scheme. Our purpose here is to examine this scheme in the context of the baryon masses.

In Sec. II we recall the necessary aspects of chiral perturbation theory and the infrared regularization scheme. Our results are given in Sec. III, first through third order and then including an estimate of fourth order. Finally our conclusions are presented in Sec. IV.

\section{THEORY}

The lowest order $S U(3)$ chiral Lagrangian [5] is

$$
\mathcal{L}^{1}=\operatorname{Tr}\left\{i \bar{B}\left(\not \partial B+[\not \mathcal{F}, B]-M_{0} \bar{B} B+\frac{1}{2} D \bar{B} \gamma_{5}\{\mu, B\}+\frac{1}{2} F \bar{B} \gamma_{5}[\psi, B]\right\}\right.
$$

Here the baryon octet is $B=2^{-\frac{1}{2}} \sum_{a=1}^{8} \lambda^{a} B^{a}$ in terms of the $S U(3)$ matrices $\lambda^{a}$ and the quantities involving the meson fields $\phi^{a}$ are

$$
\begin{aligned}
U & =u^{2}=\exp \left(\frac{i}{f} \sum_{a=1}^{8} \lambda^{a} \phi^{a}\right) \\
\Gamma_{\mu} & =\frac{1}{2}\left(u^{\dagger} \partial_{\mu} u+u \partial_{\mu} u^{\dagger}\right) ; u_{\mu}=i\left(u^{\dagger} \partial_{\mu} u-u \partial_{\mu} u^{\dagger}\right) .
\end{aligned}
$$


The second order Lagrangian is

$$
\mathcal{L}^{2}=b_{0} \operatorname{Tr}(\bar{B} B) \operatorname{Tr} \chi_{+}+b_{d} \operatorname{Tr}\left(\bar{B}\left\{\chi_{+}, B\right\}\right)+b_{f} \operatorname{Tr}\left(\bar{B}\left[\chi_{+}, B\right]\right)+\ldots,
$$

and in $\chi_{+}=2 B_{0}\left(u^{\dagger} \mathcal{M} u^{\dagger}+u \mathcal{M} u\right)$. In an obvious notation the quark mass matrix is $\mathcal{M}=$ $\operatorname{diag}\left(\hat{m}, \hat{m}, m_{s}\right)$ and this can be related to the meson masses to leading order

$$
m_{\pi}^{2}=2 \hat{m} B_{0} \quad ; \quad m_{K}^{2}=\left(\hat{m}+m_{s}\right) B_{0} \quad ; \quad m_{\eta}^{2}=\frac{1}{3}\left(4 m_{K}^{2}-m_{\pi}^{2}\right)
$$

For present purposes we can use the above Gell-Mann-Okubo relation for the $\eta$ mass; we take $m_{\pi}=0.139 \mathrm{GeV}$ and $m_{K}=0.494 \mathrm{GeV}$ yielding $m_{\eta}=0.565 \mathrm{GeV}$.

The order $Q^{2}$ contribution to the baryon masses take the familiar form

$$
\begin{aligned}
& M_{N}(2)=-2 b_{0}\left(2 m_{K}^{2}+m_{\pi}^{2}\right)-4 m_{K}^{2} b_{d}+4\left(m_{K}^{2}-m_{\pi}^{2}\right) b_{f} \\
& M_{\Lambda}(2)=-2 b_{0}\left(2 m_{K}^{2}+m_{\pi}^{2}\right)-\frac{4}{3}\left(4 m_{K}^{2}-m_{\pi}^{2}\right) b_{d} \\
& M_{\Sigma}(2)=-2 b_{0}\left(2 m_{K}^{2}+m_{\pi}^{2}\right)-4 m_{\pi}^{2} b_{d} \\
& M_{\Xi}(2)=-2 b_{0}\left(2 m_{K}^{2}+m_{\pi}^{2}\right)-4 m_{K}^{2} b_{d}+4\left(m_{\pi}^{2}-m_{K}^{2}\right) b_{f} .
\end{aligned}
$$

The order $Q^{3}$ contribution arises from the loop diagram pictured in Fig. 1 and the guts of this is the integral

$$
H=-i \mu^{4-d} \int \frac{d^{d} \ell}{(2 \pi)^{d}} \frac{1}{\left(\ell^{2}-m_{M}^{2}+i \epsilon\right)\left(2 \ell \cdot P+\ell^{2}+P^{2}-M_{B^{\prime}}^{2}+i \epsilon\right)},
$$

in dimension $d$, with $P$ denoting the four-momentum of the external baryon leg, $B$. In [4] it was argued that "hard" part of the integral, dominated by poles at momenta of the order of the baryon mass, should be absorbed in the coefficients of the effective Lagrangian. The "soft" part of the integral on the other hand needs to be calculated explicitly and can be obtained by expanding out $\ell^{2}$ from the baryon propagator and interchanging the order of integration and summation (the latter of course changes the value of the integral). In dimensional regularization the net effect is that $\ell^{2}$ is replaced by $m_{M}^{2}$ so that (6) is replaced by

$$
I=-i \mu^{4-d} \int \frac{d^{d} \ell}{(2 \pi)^{d}} \frac{1}{\left(\ell^{2}-m_{M}^{2}+i \epsilon\right)\left(2 \ell \cdot P+m_{M}^{2}+P^{2}-M_{B^{\prime}}^{2}+i \epsilon\right)}
$$


A similar result has been obtained by Becher and Leutwyler [3]. They combine the denominators in Eq. (6) using an integration over a Feynman parameter $z$. The "soft" part is then defined by extending the integration range from $0-1$ to $0-\infty$ and this includes the infrared singular contribution of leading order $Q^{d-3}$ in the chiral limit $m_{M} \rightarrow 0$ for $P^{2} \simeq M_{B^{\prime}}^{2}$. The net effect of these maneuvers is to replace the denominator $(a b)^{-1}$ in $H$ by the denominator $[a(b-a)]^{-1}$ to give $I$ in (7). These authors refer to this as infrared regularization. In heavy baryon theory the second denominator in (7) is expanded out thus leaving $(2 \ell \cdot P+i \epsilon)^{-1}$ in leading order. Becher and Leutwyler have argued against making this expansion since it breaks down in certain regions of parameter space. The present procedure can be viewed as a summation of the heavy baryon insertions in the baryon propagator to all orders. The leading order term preserves the Weinberg power counting, but in addition higher order contributions are included.

The integral that needs to be evaluated for the loop diagram of Fig. 1 is

$$
H=-i \mu^{4-d} \int \frac{d^{d} \ell}{(2 \pi)^{d}} \frac{\gamma_{5} \ell\left(\not P+\ell+M_{B^{\prime}}\right) \gamma_{5} \ell}{\left(\ell^{2}-m_{M}^{2}+i \epsilon\right)\left(2 \ell \cdot P+\ell^{2}+P^{2}-M_{B^{\prime}}^{2}+i \epsilon\right)} .
$$

We apply the so-called infrared regularization on shell, $P^{2}=M_{B}^{2}$. The resulting integrals contain ultraviolet divergences and these are removed in the standard $\overline{M S}$ scheme; this requires polynomial counterterms of arbitrarily high order in $Q$ which we do not need to specify. Then Eq. (8) becomes

$$
\begin{aligned}
I\left(M_{B}, M_{B^{\prime}}, m_{M}\right)= & \frac{\left(M_{B}^{2}-M_{B^{\prime}}^{2}\right) m_{M}^{2}}{32 \pi^{2} M_{B}} \ln \frac{m_{M}^{2}}{\mu^{2}} \\
& +\frac{\left(M_{B}+M_{B^{\prime}}\right)}{16 \pi^{2} M_{B}}\left[\left(M_{B}-M_{B^{\prime}}\right) z-m_{M}^{2}\right] J\left(z, m_{M}\right),
\end{aligned}
$$

where $z=\left(M_{B}^{2}-M_{B^{\prime}}^{2}+m_{M}^{2}\right) /\left(2 M_{B}\right)$ and

$$
J(z, m)= \begin{cases}z-z \ln \frac{m^{2}}{\mu^{2}}-2 \sqrt{m^{2}-z^{2}} \cos ^{-1}\left(-\frac{z}{m}\right) & |z|<m \\ z-z \ln \frac{m^{2}}{\mu^{2}}-\sqrt{z^{2}-m^{2}} \ln \frac{z+\sqrt{z^{2}-m^{2}}}{z-\sqrt{z^{2}-m^{2}}} & |z|>m .\end{cases}
$$

This is equivalent to the expression given in Ref. [3] and is of leading order $Q^{3}$. It is natural to identify the renormalization scale $\mu$ in this equation with the natural scale in the problem, namely the baryon mass in the chiral limit, $M_{0}$. The total third order contribution is then 
$M_{B}(3)=\sum_{B^{\prime} M} \alpha_{B B^{\prime} M} I\left(M_{B}, M_{B^{\prime}}, m_{M}\right)$, where the coefficients $\alpha$ are easily evaluated using Eq. (11) and are listed in the Appendix.

To evaluate the baryon masses in fourth order $\mathcal{L}^{2}$ requires additional terms besides those displayed in Eq. (3). They contribute to the loop integrals, but the value of the coefficients

is a priori unknown. Further, the finite parts of $\mathcal{L}^{4}$ play a role at tree level, again introducing unknown coefficients. Borasoy and Meissner [1] estimated these low energy constants. They concluded that, while their effect is not negligible, the dominant fourth order contribution arises from the meson loop diagram pictured in Fig. 2 evaluated using the three terms of $\mathcal{L}^{2}$ given in Eq. (3). Therefore we will consider just this contribution in order to get an estimate of the magnitude of fourth order. Since only loops containing baryons require special treatment in order to keep track of the chiral order, the meson tadpole diagram of Fig. 2 is evaluated in standard fashion irrespective of whether the IR or HBChPT schemes are used. For nucleons the result, once divergences have been absorbed in the counterterms, is

$$
\begin{gathered}
M_{N}(4)=\left\{3\left(2 b_{0}+b_{d}+b_{f}\right) m_{\pi}^{4} \ln \frac{m_{\pi}^{2}}{\mu^{2}}+2\left(4 b_{0}+3 b_{d}-b_{f}\right) m_{K}^{4} \ln \frac{m_{K}^{2}}{\mu^{2}}+2 b_{0} m_{\eta}^{4} \ln \frac{m_{\eta}^{2}}{\mu^{2}}\right. \\
\left.+\left(-b_{d}+\frac{5}{3} b_{f}\right) m_{\pi}^{2} m_{\eta}^{2} \ln \frac{m_{\eta}^{2}}{\mu^{2}}+\frac{8}{3}\left(b_{d}-b_{f}\right) m_{K}^{2} m_{\eta}^{2} \ln \frac{m_{\eta}^{2}}{\mu^{2}}\right\} /\left(4 \pi f^{2}\right)
\end{gathered}
$$

and, as we have remarked, we take $\mu=M_{0}$. The contributions for the remaining baryons can be read off from Ref. [1].

\section{RESULTS}

\section{A. Through Third Order}

In order to get a first comparison of (9) with the corresponding heavy baryon result we simply use the physical masses of the particles and pick a reasonable value of the renormalization scale, $\mu=1 \mathrm{GeV}$. The decay constant $f$ is taken to be the average of the kaon and pion decay constants [6], $f=0.103 \mathrm{GeV}$. For the parameters $D$ and $F$ we take the 
ratio from Close and Roberts [7] and their sum is the axial coupling constant [6], giving $D=0.804$ and $F=0.463$. The result is labelled IREX in Table 1 and can be compared with the heavy baryon case, labelled HB, for which Eq. (9) is simply replaced by $m_{M}^{3} /(8 \pi)$. The dominant effect comes from the ratio $z / m$ in Eq. (10) which is set to zero in the heavy baryon approach, but can be as large as 0.7 in magnitude here. Clearly it is largest when $B \neq B^{\prime}$ which is always the case for the kaons, since they carry strangeness, and they dominate numerically. In comparison to the heavy baryon case the net result is a rather small increase for the $N$ and $\Sigma$ and a fairly substantial, $\sim 30 \%$, reduction for the $\Lambda$ and $\Xi$.

Obviously this is not a consistent procedure and since $m_{M}^{2}$ is of $\mathcal{O}\left(Q^{2}\right)$ we should treat the baryon masses to the same order. Thus we should evaluate $M_{B}(3)=\sum_{B^{\prime} M} \alpha_{B B^{\prime} M} I\left(M_{0}+\right.$ $\left.M_{B}(2), M_{0}+M_{B^{\prime}}(2), m_{M}\right)$. The total baryon mass through third order is then $M_{B}^{\text {tot }}=$ $M_{0}+M_{B}(2)+M_{B}(3)$. Further, as we have remarked, we wish to choose the renormalization scale to be the baryon mass in the chiral limit, i.e. $\mu=M_{0}$. In order to disentangle the constants $M_{0}$ and $b_{0}$ we need a further piece of information for which we select the $\pi N$ sigma term, $\sigma_{\pi N}(0)=\hat{m} \partial M_{N}^{\text {tot }} / \partial \hat{m}$. The actual value of the sigma term is not precisely known, but for present purposes we will take the currently accepted figure of $45 \mathrm{MeV}$ from Gasser et al. [8]. This allows us to determine the unknown parameters $b_{0}, b_{d}, b_{f}$ and $M_{0}$ by performing a least squares fit to the baryon masses and $\sigma_{\pi N}(0)$. The strange quark contribution to the nucleon mass $S \equiv m_{s}\langle N|\bar{s} s| N\rangle=m_{s} \partial M_{N}^{\text {tot }} / \partial \hat{m}_{s}$ can then be calculated. We obtain $S=360 \mathrm{MeV}$. While the magnitude of $S$ is poorly known, this appears a little large so we constrained $S$ to be $200 \mathrm{MeV}$ in the final fit shown under the rubric IR in Tables 1 and 2; this degraded the accuracy of the fit to the baryon masses by about $10 \mathrm{MeV}$. Note that a positive value for $S$ is favored here, whereas the heavy baryon case [9] gives a negative value of similar magnitude. This would suggest larger $K N$ sigma terms here for which there is some weak experimental support [10]. (To obtain the parameters listed in the heavy baryon column of Table 2 the masses and $\sigma_{\pi N}(0)$ were fitted with a resulting $S=-195 \mathrm{MeV}$.)

Table 1 gives some indication of the sensitivity to the baryon masses employed. The net loop results evaluated in the IR scheme are now in all cases smaller than in the HB case 
with the reduction ranging from $9 \%$ for the $\Sigma$ to $63 \%$ for the $\Lambda$ and the bulk of the effect arises from the kaon loop contribution. Note that, referring to Eq. (10), the magnitude of the ratio $z / m$ varies over a wide range, from 0.04 to almost 2 . Thus the assumption of the heavy baryon scheme that this ratio is small, so that a power series expansion can be made, is questionable. Table 2 shows that the baryon masses are fit in the IR scheme to an accuracy $\sim 50 \mathrm{MeV}$. More relevant is the ratio of the third and second order contributions. In the HB case this ranges from 1.11 for the $N$ to 0.70 for the $\Sigma$ and $\Xi$, whereas in the IR case it ranges from 0.54 for the $\Sigma$ to 0.30 for the $\Lambda$. The convergence of the expansion is clearly better in the infrared regularization scheme, due to the smaller loop integrals. The values of $\sigma_{\pi N}(0)$ and $S$ used in the fit are uncertain. If $\sigma_{\pi N}(0)$ is increased to $55 \mathrm{MeV}$ or $S$ is increased to $300 \mathrm{MeV}$ the fit to the masses is improved slightly and the ratio of third to second order becomes slightly smaller. Conversely for a reduction of $\sigma_{\pi N}(0)$ to $35 \mathrm{MeV}$ or a reduction of $S$ to $100 \mathrm{MeV}$ the results go in the opposite direction. The changes are reasonably small so that the tabulated case is representative of our results.

\section{B. Fourth Order Estimate}

Here we estimate fourth order by considering the diagram of Fig. 2. If this is calculated using the parameters previously determined, the value of the diagram in the IR scheme is $0.17,0.21,0.24$ and $0.27 \mathrm{GeV}$ for the $N, \Lambda, \Sigma$ and $\Xi$, respectively. For the $N$ and the $\Lambda$ these contributions are similar in magnitude to third order, while for the remaining two baryons they are about half of third order. In the HB approach the renormalization scale enters for the first time in fourth order and, taking $\mu=1 \mathrm{GeV}$ as in Ref. [1], we find 0.26, 0.38, 0.40 and $0.51 \mathrm{GeV}$ for the $N, \Lambda, \Sigma$ and $\Xi$, respectively. The difference in the magnitudes in the two schemes largely reflects the different values chosen for the renormalization scale.

Since the contributions are sizeable, the parameters should be fitted as before with the fourth order contribution included. The baryon masses thus become $M_{B}^{\text {tot }}=M_{0}+M_{B}(2)+$ $M_{B}(3)+M_{B}(4)$. The results are given in Table 3 . In the IR approach the fitting produces 
two minima of similar depth. We reject the solution with a rather low mass $M_{0}$ of $0.463 \mathrm{GeV}$ and a negative value of $b_{d}$ and display the solution which appears to evolve more naturally from the third order parameterization. Here the value of $M_{0}, 0.653 \mathrm{GeV}$, is about $10 \%$ smaller than in Table 2 which causes the fourth order contributions to be reduced by about $37 \%$; the sensitivity is obviously due to the fact that $M_{0}$ is becoming comparable to the eta and kaon masses. The second and third order contributions display only modest changes from Table 2, while the totals give a little better fit to the masses here with an average deviation of $40 \mathrm{MeV}$. In the $\mathrm{HB}$ case the results displayed correspond to a renormalization scale $\mu=1 \mathrm{GeV}$; they show qualitatively the same trends as the complete fourth order calculation of Borasoy and Meissner [1]. The alternative of choosing the renormalization scale self-consistently results in $\mu=M_{0}=0.868 \mathrm{GeV}$ and the fourth order values of Table 3 are decreased by 17\%, while second order shows a small $5 \%$ increase. Either way the strong cancellation, and in some cases overcancellation, between second and third order remains. In the IR case however the magnitude of third order is about half that of second order, and our fourth order estimate is smaller on average by roughly a further factor of a half.

It is also interesting to examine the order-by-order contributions to the sigma term

$$
\begin{gathered}
\sigma_{\pi N}^{I R}=0.074-0.036+0.008=0.045 \mathrm{GeV} \\
\sigma_{\pi N}^{H B}=0.059-0.032+0.018=0.045 \mathrm{GeV} .
\end{gathered}
$$

Our values for $\sigma_{\pi N}^{H B}$ are quite close to those reported by Borasoy and Meissner [1]. The convergence of the series for $\sigma_{\pi N}$ is similar in the two schemes, with the IR approach weakly favored. For completeness we also give breakdown of the strange quark contribution to the nucleon mass, while noting that $S$ was fitted in the IR calculation but left free in the HB case,

$$
\begin{aligned}
S^{I R} & =0.254-0.059+0.005=0.200 \mathrm{GeV} \\
S^{H B} & =0.093-0.346+0.230=-0.023 \mathrm{GeV} .
\end{aligned}
$$

While the behavior of the IR series looks much better, we caution that the very small value 
of the fourth order contribution is somewhat fortuitous since it is very sensitive to the value of the scale $\mu$.

\section{CONCLUSIONS}

In conclusion we have examined the numerical implications of a new form of $S U(3)$ chiral perturbation theory where the loop integrals are evaluated using the so-called infrared regularization scheme. We have examined the chiral series through third order and made an estimate of fourth order by evaluating the dominant contribution. The most important feature is that the magnitude of the third order loop integral contribution to the baryon octet masses is smaller in the infrared scheme than in HBChPT. This means that the strong cancellation, in some cases overcancellation, of second order that is characteristic of HBChPT no longer occurs. Thus the convergence of the chiral series appears to be better when infrared regularization is used with successive terms decreasing in magnitude by about a factor of a half. Given that the ratio of the kaon and eta masses to the baryon masses is of this order, this is probably the most that one could expect.

\section{ACKNOWLEDGEMENTS}

We thank the referee for a useful suggestion. This work was supported in part by the US Department of Energy under grant DE-FG02-87ER40328.

\section{APPENDIX}

We list here the non-zero coefficients $\alpha$ needed in the evaluation of Fig. 1.

$$
\begin{aligned}
& \alpha_{N N \pi}=\alpha_{\Xi \Sigma K}=\frac{3}{2} \alpha_{\Sigma \Xi K}=-\frac{3(D+F)^{2}}{4 f^{2}} \\
& \alpha_{N \Sigma K}=\alpha_{\Xi \Xi \pi}=\frac{3}{2} \alpha_{\Sigma N K}=-\frac{3(D-F)^{2}}{4 f^{2}} \\
& \alpha_{N N \eta}=\alpha_{\Xi \Lambda K}=\frac{1}{2} \alpha_{\Lambda \Xi K}=-\frac{(D-3 F)^{2}}{12 f^{2}}
\end{aligned}
$$




$$
\begin{aligned}
& \alpha_{N \Lambda K}=\alpha_{\Xi \Xi \eta}=\frac{1}{2} \alpha_{\Lambda N K}=-\frac{(D+3 F)^{2}}{12 f^{2}} \\
& \alpha_{\Lambda \Lambda \eta}=\alpha_{\Sigma \Sigma \eta}=\alpha_{\Sigma \Lambda \pi}=\frac{1}{3} \alpha_{\Lambda \Sigma \pi}=-\frac{D^{2}}{3 f^{2}} \\
& \alpha_{\Sigma \Sigma \pi}=-\frac{2 F^{2}}{f^{2}}
\end{aligned}
$$




\section{REFERENCES}

[1] B. Borasoy and U.-G. Meissner, Ann. Phys. (NY) 254, 192 (1997).

[2] J.F. Donoghue and B.R. Holstein, Phys. Lett. B 436, 331 (1998);J.F. Donoghue, B.R. Holstein and B. Borasoy, Phys. Rev. D 59, 036002 (1999).

[3] T. Becher and H. Leutwyler, Eur. Phys. J. C 9, 643 (1999).

[4] P.J. Ellis and H.-B. Tang, Phys. Rev. C 57, 3356 (1998).

[5] A. Krause, Helv. Phys. Acta 63, 3 (1990).

[6] Particle data group, Eur. Phys. J. C 3, 1 (1998).

[7] F.E. Close and R.G. Roberts, Phys. Lett. B 316, 165 (1993).

[8] J. Gasser, H. Leutwyler and M.E. Sainio, Phys. Lett. B 253, 252 (1991).

[9] V. Bernard, N. Kaiser and U.-G. Meissner, Z. Physik C 60, 111 (1993).

[10] P.M. Gensini, LNF Spring School in Nuclear and Subnuclear Physics (Frascati, Italy, 1998), hep-ph/9804344. 
Table 1

Meson contributions to the loop result for the baryon masses (in $\mathrm{GeV}$ ).

\begin{tabular}{|c|c|c|c|}
\hline & Case & $K$ & Total \\
\hline \multirow[t]{3}{*}{$N$} & $\mathrm{HB}$ & $-0.012-0.221-0.019$ & -0.252 \\
\hline & IREX & $-0.011-0.249-0.018$ & -0.278 \\
\hline & IR & $-0.012-0.129-0.019$ & -0.160 \\
\hline \multirow[t]{3}{*}{$\Lambda$} & $\mathrm{HB}$ & $-0.007-0.388-0.146$ & -0.540 \\
\hline & IREX & $-0.009-0.195-0.139$ & -0.342 \\
\hline & IR & $0.015-0.069-0.147$ & -0.201 \\
\hline \multirow[t]{3}{*}{$\Sigma$} & $\mathrm{HB}$ & $-0.006-0.389-0.146$ & -0.541 \\
\hline & IREX & $-0.004-0.406-0.140$ & -0.550 \\
\hline & IR & $-0.008-0.339-0.147$ & -0.494 \\
\hline \multirow[t]{3}{*}{$\Xi$} & $\mathrm{HB}$ & $-0.001-0.557-0.271$ & -0.829 \\
\hline & IREX & $-0.001-0.359-0.262$ & -0.622 \\
\hline & IR & $-0.001-0.314-0.274$ & -0.589 \\
\hline
\end{tabular}




\section{Table 2}

Comparison of parameters and baryon masses obtained in the heavy baryon and the present approach to third order.

\begin{tabular}{|l|rr|}
\hline Parameters & HB & IR \\
\hline$b_{0}\left(\mathrm{GeV}^{-1}\right)$ & -0.762 & -0.921 \\
$b_{d}\left(\mathrm{GeV}^{-1}\right)$ & 0.067 & 0.206 \\
$b_{f}\left(\mathrm{GeV}^{-1}\right)$ & -0.533 & -0.435 \\
$M_{0}(\mathrm{GeV})$ & 0.965 & 0.733 \\
\hline Masses $(\mathrm{GeV})$ & & \\
\hline second & 0.228 & 0.342 \\
$N$ third & -0.252 & -0.160 \\
total & 0.941 & 0.915 \\
\hline second & 0.687 & 0.671 \\
$\Lambda$ third & -0.540 & -0.201 \\
total & 1.112 & 1.204 \\
\hline second & 0.768 & 0.919 \\
$\Sigma$ third & -0.541 & -0.494 \\
total & 1.191 & 1.158 \\
\hline second & 1.187 & 1.124 \\
$\Xi$ third & -0.829 & -0.589 \\
total & 1.322 & 1.268 \\
\hline
\end{tabular}




\section{Table 3}

Comparison of parameters and baryon masses obtained in the heavy baryon and the present approach to fourth order.

\begin{tabular}{|c|c|}
\hline Parameters & $\mathrm{HB}$ \\
\hline$b_{0}\left(\mathrm{GeV}^{-1}\right)$ & $-0.577-0.830$ \\
\hline$b_{d}\left(\mathrm{GeV}^{-1}\right)$ & $0.054 \quad 0.159$ \\
\hline$b_{f}\left(\mathrm{GeV}^{-1}\right)$ & $-0.424-0.401$ \\
\hline$M_{0}(\mathrm{GeV})$ & $0.849 \quad 0.653$ \\
\hline \multicolumn{2}{|l|}{ Masses $(\mathrm{GeV})$} \\
\hline second & $0.152 \quad 0.327$ \\
\hline$N$ third & $-0.252-0.168$ \\
\hline fourth & $0.192 \quad 0.111$ \\
\hline total & $0.941 \quad 0.924$ \\
\hline second & $0.517 \quad 0.640$ \\
\hline$\Lambda$ third & $-0.540-0.246$ \\
\hline fourth & $0.287 \quad 0.136$ \\
\hline total & $1.112 \quad 1.184$ \\
\hline second & $0.581 \quad 0.830$ \\
\hline$\Sigma$ third & $-0.541-0.476$ \\
\hline fourth & $0.303 \quad 0.152$ \\
\hline total & $\begin{array}{ll}1.191 & 1.160\end{array}$ \\
\hline second & $0.914 \quad 1.048$ \\
\hline$\Xi$ third & $-0.829-0.591$ \\
\hline fourth & $0.389 \quad 0.169$ \\
\hline total & $\begin{array}{ll}1.322 & 1.280\end{array}$ \\
\hline
\end{tabular}




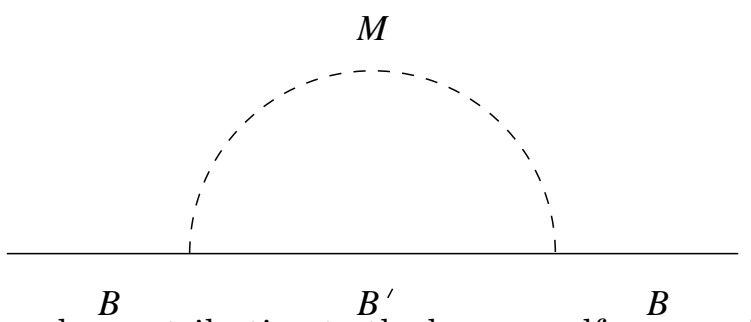

FIG. 1. One-loop third-order contribution to the baryon self-energy. The dashed line represents a meson propagator and the baryons are denoted by solid lines.

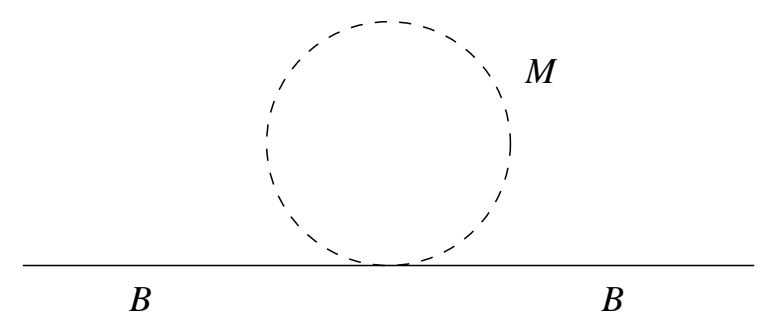

FIG. 2. One-loop fourth-order contribution to the baryon self-energy; notation as for Fig. 1. 